Article

\title{
Deduction of Lorentz Transformations from Classical Thermodynamics
}

\author{
Angela M. Ares de Parga, Fernado Angulo-Brown and Gonzalo Ares de Parga * \\ Department of Physics, School of Physics and Mathematics, National Polytechnic Institute, \\ U.P. Adolfo López Mateos, Zacatenco, C.P. 07738, Mexico D.F., Mexico; \\ E-Mails: tuo882_2@hotmail.com (A.M.A.D.P.); angulo@esfm.ipn.mx (F.A.-B.) \\ * Author to whom correspondence should be addressed; E-Mail: gadpau@ hotmail.com; \\ Tel.: +52-55-5729-6000 (ext. 55317); Fax: +52-55-5729-6000 (ext. 55015).
}

Academic Editor: Kevin H. Knuth

Received: 3 October 2014 / Accepted: 6 January 2015 / Published: 8 January 2015

\begin{abstract}
The Lorentz transformations are obtained by assuming that the laws of classical thermodynamics are invariant under changes of inertial reference frames. As Maxwell equations are used in order to deduce a wave equation that shows the constancy of the speed of light, by means of the laws of classical thermodynamics, the invariance of the Carnot cycle is deduced under reference frame changes. Starting with this result and the blackbody particle number density in a rest frame, the Lorentz transformations are obtained. A discussion about the universality of classical thermodynamics is given.
\end{abstract}

Keywords: Lorentz transformations; Carnot efficiency; laws of classical thermodynamics; invariance

\section{Introduction}

During the second part of the last century, the theory of relativistic transformation laws of the thermodynamical quantities was analyzed from different points of view. The Einstein-Planck theory [1,2] was accepted until, in the middle of the century, Einstein let his own doubts about the validity of the model be known in a personal communication to van Laue [3,4]. In the following years, countless articles appeared in the literature. Among other proposals, the works by van Kampen [5], Israel [6,7], Balescu [8], Staruszkiewicz [9], Ott [10], Arzeliès [11], Möller [12], Przanowski [13], Landsberg [14] and Rohrlich [15] must be cited. Since many of the different theories were at odds with 
each other, Balescu [8], by using statistical mechanics, showed that many of them can be reached from the Einstein-Planck proposal by means of a kind of gauge theory. Moreover, in 1998, Sieniutycz [16] summarized the three more common and acceptable relativistic transformations of the temperature as $T=\gamma^{d} T_{o}$, where $\gamma=\left(1-v^{2} / c^{2}\right)^{-1 / 2}$, being $d=1,-1$ or 0 , depending on the Ott-like proposal [10], on the Einstein-Planck-like proposal [1,2] and on the Landsberg-like proposal [14], respectively. So far this century, the never-ending discussion about the relativistic transformation laws of the thermodynamical quantities has been enriched by many authors. The idea that it is impossible to define a temperature in a moving frame was introduced by Landsberg and Matsas [4,17]. However, based on the Unrhu detector and on the fact that the distribution function of a moving blackbody does not have to coincide with the distribution function of it at rest, Ares de Parga et al. [18] showed that it is possible to define a temperature. Furthermore, by rescuing the Fermi ideas about the good definition of a tensor in special relativity, Nakamura [19,20] was able to give a covariant definition of the volume, the temperature and other thermodynamical quantities. In this order of ideas, Ares de Parga et al. [21,22], developed a consistent covariant theory, based on their first article [18], where they redefined the concept of internal energy and obtained a general theory that recovers the Rohrlich [15] and Gamba formulations [23]. However, many other articles differ from these points of view. Another line of thinking has been led by the Möller detailed analysis [12] of the covariance. In particular, he pointed out why work and internal energy cannot transform in a covariant form, whereas this is the case for heat. Moreover, the debate launched by Kibble [24], Arzeliès [25] and Gamba [26] represents the discord about the covariance of the theory. The convenience of using the four-vector volume, the so-called covariant method, is the point of contention. An interesting article in favor of Möller and Kibble was given by Requardt [27]. Nevertheless, the discussion on relativistic thermodynamics cannot be reduced just to two points of view. Actually, since the mid-twentieth century to the present, several doubts have emerged about the theory. As for example, in 1966, an interesting paradox was presented by Noerdlinger [28] about Landsberg's proposal [14], which consists of noticing that an observer in front of a moving blackbody will see a blue-shift and one behind will see a red-shift. This problem opens the door to understanding the importance of the conceptual meaning of the volume and the simultaneity in the theory. The blue-shift and the red-shift exist as described above, but the transformation law of the temperature will just depend on the factor $\sqrt{1-v^{2} / c^{2}}$. This is explained by understanding the role of the directional temperature (see Subsection 3.2).

On the other hand, it is a well-known fact that Einstein proposed that the laws of the universe are invariant in each inertial reference frame. Particularly, the well-known result on the invariance of the value of the velocity of light $c$ can be deduced from Maxwell equations. Starting from this result, he was able to deduce the Lorentz transformations and to understand the dynamics of special relativity. By using special relativity, Einstein [1] and, separately, Planck [2] set the basis for the relativistic transformation laws of the thermodynamical quantities. However, remembering the admirable statement of Einstein about thermodynamics [29], "A theory is the more impressive the greater the simplicity of its premises is, the more different kinds of things it relates, and the more extended is its area of applicability. Therefore the deep impression which classical thermodynamics made upon me. It is the only physical theory of universal content concerning which I am convinced that within the framework of the applicability of its basic concepts, it will never be overthrown", we can ask ourselves if the Lorentz 
transformations can be deduced by just considering the invariance of the laws of thermodynamics in each inertial reference frame. Therefore, the objective of this work consists of deducing the Lorentz transformations by considering that the laws of classical thermodynamics are invariant under changes of inertial reference frames. The fact of supposing the invariance of the laws of thermodynamics, as scalar equations, will lead to an Einstein-Planck-like theory and also to the Lorentz transformations. However, it does not mean that the other theories that do not come from considering such invariance have to be discarded. The present article does not attempt to defend or not the covariant method (this has been done by many authors [15,19-23]). Blackbody radiation will be used as a tool in order to obtain the final result.

The article is organized as follows: In Section 2, proposing the invariance of the classical thermodynamics laws, the invariance of the entropy and the Carnot efficiency are analyzed. The invariance of any efficiency for any type of reversible thermal cycle is demonstrated. By using the blackbody particle density and supposing the conservation of the number of particles under changes of reference frames and also the homogeneity and isotropy of the space-time, the Lorentz transformations are obtained in Section 3. In this section, a discussion of the compatibility of classical thermodynamics and the theory of general relativity is analyzed. In Section 4, a comparison between the results obtained in the previous sections and the covariant theory developed by Ares de Parga et al. [18,21,22] is made. Finally, in Section 5, some concluding remarks about the universality of classical thermodynamics are exposed.

\section{Invariance of Thermodynamics}

\subsection{Invariance of the Entropy and the Efficiency of the Carnot Cycle}

Let us define a reference frame as the one where the laws of classical thermodynamics are valid, and suppose that in any other reference frame that possesses a constant velocity with respect to the first one, the laws of thermodynamics are also valid; that is, it has been assumed that the laws of thermodynamics are invariant under changes of inertial reference frames. Our objective consists of investigating if this assumption gives us some information about the transformation laws of the space-time; that is: if an event in an inertial reference frame $K_{o}$ is described by $\left(t_{o}, x_{o}, y_{o}, z_{o}\right)$, which event $(t, x, y, z)$ will correspond in another inertial reference frame $K$,

$$
(t, x, y, z)=\left(t\left(t_{o}, x_{o}, y_{o}, z_{o}\right), x\left(t_{o}, x_{o}, y_{o}, z_{o}\right), y\left(t_{o}, x_{o}, y_{o}, z_{o}\right), z\left(t_{o}, x_{o}, y_{o}, z_{o}\right)\right)
$$

From the second law of thermodynamics, it is well known that the entropy can be related with the number of microstates of a thermodynamical system. Since the number of microstates must be an invariant in any inertial reference frame,

$$
d S=\frac{\delta Q_{o}}{T_{o}}=\frac{\delta Q}{T}
$$

where $\delta Q_{o}, T_{o}, \delta Q$ and $T$ represent the heat and the temperature in $K$ and $K_{o}$, respectively. Immediately, it can be inferred that the transformation laws of the heat and the temperature are linear; that is:

$$
\delta Q=a \delta Q_{o} \quad \text { and } \quad T=a T_{o} .
$$


It has to be noted that by assuming homogeneity of the space, the parameter " $a$ " can be considered independent of the event. Therefore, considering $a \neq a\left(t_{o}, x_{o}\right)$, gravitational effects are not taken into account, and the theory is constrained to special relativity. Let us consider a Carnot cycle in $K_{o}$. The first step to prove is that a Carnot cycle in $K_{o}$ also corresponds to a Carnot cycle in $K$. Thus, it is necessary to show that an adiabatic and an isothermal path transform into an adiabatic and an isothermal path, respectively. Since in an adiabatic path in $K_{o}$, the heat vanishes, $\delta Q_{o}=0$, then in $K$, due to Equation (3), the heat also vanishes, $\delta Q=0$. In the case of an isothermal path, the temperature is kept constant, $T_{o}=$ constant; therefore, due to Equation (3), the temperature is also a constant in $K$, $T=$ constant. It can be concluded that a Carnot cycle in $K_{o}$ is also a Carnot cycle in $K$. The efficiency in both frames can be calculated,

$$
\eta_{o}=1-\frac{T_{o 1}}{T_{o 2}}=1-\frac{T_{1}}{T_{2}}=\eta .
$$

Thus, the invariance of the Carnot cycle is shown. Here, it is convenient to make a comment. As in electrodynamics, Maxwell equations are supposed to be invariant in all of the inertial reference frames, and then, a wave equation can be deduced, proving the invariance of the speed of light; in thermodynamics, by assuming the invariance of the their laws, the invariance of the efficiency of the Carnot cycle is deduced.

\subsection{Relativistic Transformations of the Internal Energy and the Work}

Let us begin by citing a comment given by Requardt [27], "Heat is a subtle concept even in non-relativistic phenomenological thermodynamics. The most straightforward way of introducing it is, in our view, to regard it as the stochastic, disordered and non-coherent contribution in the energy conservation law (in contrast to, e.g., the highly organized form of work, which is, in effect, some averaged and integrated form of the individual effects of many microscopic events). So it is reasonable to define it simply by $\delta Q=d U+\delta W$. That is, as the difference between the increase of internal energy and applied work". Since we are supposing the invariance of classical thermodynamics, the first law must be written as usual:

$$
\Delta U=Q-W
$$

Even if we can doubt the validity of our main assumption, we are obligated to consider the first law as the definition of heat in order to be consistent. The consequences of such a definition are exposed in Section 4, where the meaning of internal heat will be redefined. It can be concluded that the difference between the increment of the internal energy and the work must transform as the heat; that is:

$$
\Delta U+W=Q=a Q_{o}=a\left(\Delta U_{o}+W_{o}\right)
$$

Previously, it has been proven that the Carnot efficiency cycle is an invariant. This means that $W / Q$ is also an invariant. Therefore, it can be concluded that:

$$
U=a U_{o} \quad \text { and } \quad W=a W_{o} .
$$

This is surprising, since when a regular deduction of the work transformation is done, as for example in Tolman [30], a momentum term appears. However, it was proven by Ares de Parga et al. [18,21,22] that 
in relativistic thermodynamics, the concept of internal energy and work have to be redefined. This point will be expanded in the discussion section.

\subsection{Invariance of the Efficiency of Any Cycle}

In 1991, Angulo-Brown [31] defined the $g$-function, and he demonstrated that for any endoreversible cycle performing between two temperatures $T_{o 1}$ and $T_{o 2}$, the power output $\Pi_{o}$ is related with the production of entropy $\sigma_{o}$ by:

$$
\Pi_{o}=g_{o} \sigma_{o}
$$

where $g_{o}$ is given by:

$$
g_{o}=\frac{T_{o 1} T_{o 2}}{T_{o 1}-T_{o 2}-\eta_{o} T_{o 1}} .
$$

This relation is obtained by using the first and second laws of thermodynamics. Therefore, it must be valid in any inertial reference frame; that is:

$$
\Pi=g \sigma
$$

Or simply:

$$
d W=g d S=a d W_{o}=a g_{o} d S
$$

Therefore,

$$
g=a g_{o}
$$

Taking into account Equation (9), the only way to reach Equation (12) consists of having:

$$
\eta=\eta_{o}
$$

for any type of reversible cycle. It can be concluded that not only the Carnot efficiency is an invariant, but any thermodynamical cycle efficiency is an invariant, too.

An interesting point can be mentioned. If we had assumed at the beginning that the efficiency of the Carnot cycle is an invariant, we would have obtained that the entropy is an invariant. That is,

$$
\eta=1-\frac{T_{1}}{T_{2}}=\eta_{o}=1-\frac{T_{o 1}}{T_{o 2}} \Rightarrow T=a T_{o}
$$

and:

$$
\eta=\frac{W}{Q_{2}}=1-\frac{Q_{1}}{Q_{2}}=1-\frac{Q_{o 1}}{Q_{o 2}}=\eta_{o} \Rightarrow Q=b Q_{o}
$$

By reviewing the definition of the scale of temperatures, it is obvious that $a=b$. Therefore,

$$
d S=\frac{\delta Q}{T}=\frac{\delta Q_{o}}{T_{o}}=d S_{o}
$$




\section{Lorentz Transformations}

\subsection{Pressure-Volume Transformation}

So far, just thermodynamical quantities have been studied in order to know their transformation laws. Nevertheless, the objective consists of deducing the space transformations. By using the first law for a PVT system, the volume transformation can be approached, since:

$$
d U=\delta Q-P d V
$$

therefore, it is clear that:

$$
P d V=a P_{o} d V_{o}
$$

It has been deduced that the product of the pressure and volume transforms as the heat, but within a thermodynamic context, it is impossible to obtain a transformation law for the pressure or the volume individually. That is, let us consider the Helmholtz free energy:

$$
F=U-T S
$$

It is well-known that,

$$
P=-\left[\frac{\partial F}{\partial V}\right]_{T}
$$

From Equation (19), it is easy to see that $F$ transforms as the heat. Once again, just the product of the pressure and the volume transforms as the heat. At this stage, it is necessary that something is more in order to know the transformation of the volume. Therefore, we can put:

$$
P=\epsilon P_{o} \quad \text { and } \quad V=\kappa V_{o},
$$

where $\epsilon$ and $\kappa$ depend on the velocity $v$ and they are such that:

$$
\epsilon \kappa=a .
$$

It is interesting to note that classical thermodynamics is compatible with a more general theory of dynamics and transformation laws of the space-time. Indeed, Newtonian dynamics (with Galilean transformations) and relativistic dynamics (with Lorentz transformations) both imply that the pressure is an invariant. Nevertheless, due to Equations (21) and (22), classical thermodynamics allows the pressure to transform differently. We will continue considering that the pressure is not an invariant until we find a reason to deny it.

\subsection{Homogeneity, Isotropy and Smoothness of the Transformations}

The assumption of the homogeneity and isotropy of the space not only permits us to assume that the parameter " $a$ " is independent of the space-time coordinates, but it also allows us to explore other physical properties. 


\subsubsection{Particle Density of Blackbody}

If a blackbody is considered, the density of particles can be deduced by using statistical mechanics [32,33]. No relativity has been used, but just quantum mechanics and the fact that each photon moves with a speed equal to light speed $c$ in the reference frame at rest.

$$
n_{o}\left(w, T_{o}\right) d w d \Omega=\frac{w^{2} / c^{3}}{\pi^{2}\left[\left(\exp \frac{\hbar w}{k T_{o}}\right)-1\right]} d w d \Omega,
$$

where the solid angle has been introduced in order to anticipate the form of the expression on a moving system. The question now is to deduce the particle density $n$ of a moving blackbody with speed $v$ with the following properties:

(A) The particle density $n$ in the moving frame must coincide with $n_{o}$, the particle density at rest, when the speed $v$ tends to zero; that is,

$$
\lim _{v \rightarrow 0} n(w, T)=n_{o}\left(w, T_{o}\right) .
$$

(B) The number of particles in the volume must be the same, that is [32],

$$
N=V \iint n(w, T) d w d \Omega=V_{o} \iint n_{o}\left(w, T_{o}\right) d w d \Omega=V_{o} \frac{4 \xi(3)}{\pi}\left(\frac{k T_{o}}{\hbar c}\right)^{3} .
$$

(C) The transformation of the temperature must be included:

$$
n(w, T)=n\left(w, a T_{o}\right) .
$$

(D) The parameter " $a$ " tends to unity in the limit of $v$ tending to zero (this is related to the homogeneity of the space-time),

$$
\lim _{v \rightarrow 0} a=1 .
$$

(E) The frequency, in the non-relativistic Doppler effect, changes as:

$$
w^{\prime}=\frac{w}{1-v / c \cos \theta} .
$$

Therefore, to be consistent with the non-relativistic limit as in A, it will be necessary that the particle density depends on $v / c(\cos \theta)$, that is,

$$
n=n\left(w, a T_{o}, v / c \cos \theta\right)
$$

This is related to the isotropy of the space.

Finally, in order to accomplish the above five conditions, we propose:

$$
n d w d \Omega=\frac{w^{2} / c^{3}}{\pi^{2}\left[\left(\exp \frac{\hbar w}{k a T_{o} /(1-v / c \cos \theta)}\right)-1\right]} d w d \Omega .
$$

Properties A, C, D and E are fulfilled by using the expression for the particle density in Equation (30). It is necessary to use Property $\mathrm{B}$, in order to deduce the value of $a$. Integrating Equation (30) over the solid angle, one obtains:

$$
\int n\left(w, a T_{o}, v / c \cos \theta\right) d \Omega=\frac{k a T_{o} w}{\pi v \hbar c^{2}} \ln \left\{\frac{1-\exp \frac{\hbar w(1+v / c)}{k T_{o} a}}{1-\exp \frac{\hbar w(1-v / c)}{k T_{o} a}}\right\} .
$$


Achieving the final integration over the frequencies arrives at,

$$
\int n\left(w, a T_{o}, v / c \cos \theta\right) d \Omega d w=\gamma^{4} \frac{4 \xi(3)}{\pi}\left(\frac{k a T_{o}}{\hbar c}\right)^{3} .
$$

where $\xi$ represents the zeta function. Therefore, the number of particles is:

$$
N=V \gamma^{4} \frac{4 \xi(3)}{\pi}\left(\frac{k a T_{o}}{\hbar c}\right)^{3}=\kappa V_{o} \gamma^{4} \frac{4 \xi(3)}{\pi}\left(\frac{k a T_{o}}{\hbar c}\right)^{3} .
$$

Comparing Equations (33) and (30), it can be concluded that:

$$
\kappa \gamma^{4} a^{3}=1 .
$$

Until now, it is impossible to deduce the value of $a, \kappa$ and $\epsilon$. However, due to the homogeneity of the space, it can be inferred that $a, \kappa$ and $\epsilon$ are functions just of the velocity between the reference frames and not of the space; that is:

$$
a=a(v), \quad \kappa=\kappa(v) \quad \text { and } \quad \epsilon=\epsilon(v) .
$$

\subsubsection{Symmetries}

From the Einstein postulate of invariance of the laws of nature, without using classical electrodynamics, Terletskii [34] has shown that a general linear transformation between two sets of space-time coordinates must be of the Lorentz form if the inverse and products of all transformations have the appropriate structure related to the symmetry properties already supposed. Moreover, recently, Mermin [35] deduced Lorentz-like transformations with a general constant $b$, which does not have to coincide with the speed of light. In this order of ideas, let us deduce the type of transformations by using Equations (21) and (35). First of all, if we consider a transformation between two reference frames in one direction, that is, $\vec{v}=v \hat{i}$, that is in the $x$-axis, the transformations of the axes $y$ and $z$ must be independent of the time by homogeneity of the time; that is:

$$
d y=\lambda d y_{o} \quad \text { and } \quad d z=\rho d z_{o},
$$

where $\lambda$ and $\rho$ are functions of the relative velocity between the two reference frames. By symmetry, the transformation from $K$ to $K_{o}$ must be the same,

$$
d y_{o}=\lambda d y \quad \text { and } \quad d z_{o}=\rho d z .
$$

This obviously means that:

$$
\lambda=\rho=1 .
$$

Therefore,

$$
y=y_{o} \quad \text { and } \quad z=z_{o} .
$$

The fact that the volume transforms as in Equation (21) and by using the assumption of the homogeneity of the space-time imply that the transformation law for the $x$ and $t$ coordinates must be of the form:

$$
\begin{aligned}
x_{o} & =\kappa^{-1}(x-\beta b t) \\
b t_{o} & =\kappa^{-1}(b t-\beta x)
\end{aligned}
$$


where $b$ represents a parameter whose units are of a speed and $\beta$ another parameter without units and depending just on the speed. By considering the symmetries, it is clear that the inverse transformations are:

$$
\begin{aligned}
x & =\kappa^{-1}\left(x_{o}+\beta b t_{o}\right) \\
b t & =\kappa^{-1}\left(b t_{o}+\beta x_{o}\right)
\end{aligned}
$$

By making a systematic application of the transformations, we arrive at:

$$
\begin{aligned}
x & =\kappa^{-1}\left(x_{o}+\beta b t_{o}\right)=\kappa^{-1}\left(\kappa^{-1}(x-\beta b t)+\beta \kappa^{-1}(b t-\beta x)\right) \\
& =\kappa^{-2}\left(1-\beta^{2}\right) x+\kappa^{-2}(-\beta+\beta) b t .
\end{aligned}
$$

Therefore, it can be concluded that:

$$
\kappa^{2}=\left(1-\beta^{2}\right)
$$

However, $\beta$ represents just a factor that is a function of $v / b$. To know its functionality, it is necessary to note that an invariant can be built:

$$
\begin{aligned}
d s^{2} & =b^{2} d t_{o}^{2}-d x_{o}^{2} \\
& =\kappa^{-2}(b d t-\beta d x)^{2}-\kappa^{-2}(x-\beta b t)^{2} \\
& =\kappa^{-2}\left(1-\beta^{2}\right)\left(b^{2} d t^{2}-d x^{2}\right)=b^{2} d t^{2}-d x^{2}
\end{aligned}
$$

This means that if the particle is at rest in a moving frame, an inertial frame, with constant speed $v$ with respect to $K_{o}$, we can define a proper time $d \tau$,

$$
d \tau=\frac{d s}{b}=d t_{o} \sqrt{1-\frac{v^{2}}{b^{2}}}
$$

Finally, we can assert that $\beta$ can be identified with:

$$
\beta=\frac{v}{b}
$$

Therefore, we arrived at the same result found by Mermin [35], and the parameter $\kappa$ is equal to:

$$
\kappa=\sqrt{1-\frac{v^{2}}{b^{2}}},
$$

where $b$ represents a constant quantity with speed unities.

\subsubsection{Dynamics}

Once the transformation laws have been proven to be of the Lorentz form, it is easy to see that the dynamics is equivalent to the one presented in special relativity, but with $b$ instead of $c$. First of all, If we consider a transformation between two reference frames in one direction, that is, $\vec{v}=\widehat{v i}$, which is in the $x$-axis, the force in the $x$-axis is such that:

$$
F_{x}=F_{x o} .
$$


The area perpendicular to the $x$-axis transforms as an invariant,

$$
A_{x}=A_{x o} .
$$

Finally, the pressure transforms as an invariant, that is:

$$
P=\frac{F_{x}}{A_{x}}=\frac{F_{x o}}{A_{x o}}=P_{o}
$$

It can be concluded that:

$$
\epsilon=1 \text {. }
$$

Therefore,

$$
\kappa=a
$$

Equation (34) is now:

$$
a^{4} \gamma^{4}=1
$$

We arrive at:

$$
a=\sqrt{1-\frac{v^{2}}{c^{2}}} \quad \text { and } \quad b=c .
$$

Finally, by using the symmetry properties, the Lorentz transformations have been obtained.

Therefore, the particle density of a moving blackbody can be expressed as:

$$
n=\frac{w^{2} / c^{3}}{\pi^{2}\left[\left(\exp \frac{\hbar w}{k T /(1-v / c \cos \theta)}\right)-1\right]}=\frac{w^{2} / c^{3}}{\pi^{2}\left[\left(\exp \frac{\hbar w}{k \sqrt{1-v^{2} / c^{2}} T_{o} /(1-v / c \cos \theta)}\right)-1\right]} .
$$

Even if this result has been deduced by using some heuristic assumptions (Properties A to E), it coincides with the expression due to Henry et al. [36], Peebles and Wilkinson [37] (some factors are different due to the flux character or the used units; here, we are following Greiner spatial density of photons [33]). For obtaining the Bracewell-Conklin relation [38], the energy spectrum, Equation (55) must be multiplied by $\hbar w$. The directional temperature is included in the result in the exponential term as:

$$
T_{d i r}=\frac{T_{o}}{\frac{1-v / c \cos \theta}{\sqrt{1-v^{2} / c^{2}}}}
$$

where the minus sign comes from following the Landsberg convention [4,17], which indicates that the rest frame of the blackbody is moving to the right of the laboratory frame. It is surprising that by using heuristic assumptions, the experimental Bracewell-Conklin result is obtained. The directional temperature appears just as an experimental parameter, but the temperature of the moving blackbody in this model corresponds to:

$$
T=\gamma^{-1} T_{o}
$$




\subsection{Non-linear Transformations}

The Lorentz transformations have been obtained by using the homogeneity and isotropy of the space-time. However, if these properties are not considered, we cannot assume that $a=\sqrt{1-\frac{v^{2}}{c^{2}}}$. In general relativity, which implies the non-homogeneity of the space-time in the presence of mass, if we consider an accelerated reference frame, which is equivalent to the presence of a gravitational field, the energy and the temperature have to be redefined in order to achieve the meaning of the entropy. The entropy depends on the internal microstates of a body. It cannot change due to the presence of a gravitational field. Consequently, since the temperature is obtained by deriving the energy with respect to the entropy, the energy and the temperature in a non-comoving system must be redefined as [32]:

$$
E=E_{o} \sqrt{-g_{o o}} \quad \text { and } \quad T=T_{o} \sqrt{-g_{o o}},
$$

where $g_{o o}$ represents the "oo" component of the metric tensor. This means that the transformation parameters $a$ and $\kappa$ are such that:

$$
\kappa \neq a
$$

as it is accepted by classical thermodynamics. Of course, in the deduction of the particle density, an isotropy of the space has been assumed, a fact that does not happens in general relativity, and therefore, the proposal in Equation (30) is not any more valid in this case. The conclusion is that classical thermodynamics accepts other kind of dynamics, in particular by redefining some quantities, it is compatible with general relativity. Moreover, by assuming that the entropy is proportional to the area of a causal horizon, Jacobson [39] derived the Einstein equation. This equation can be seen as an equation of state. The universality of classical thermodynamics has been shown one more time. However, Jacobson deduction is based on using Unrhu effect, boost transformations, Killing vectors and many tools coming from relativity. Our deduction of the Lorentz transformations is obtained by just considering the invariance of the laws of thermodynamics and some assumptions about the symmetry of the time-space (Properties A to E). In the process, the Bracewell-Conklin experimental result is found.

\section{Discussion}

In order to understand the transformation laws of the thermodynamical quantities described above, some of them must be redefined. If we use the regular or mechanical definition of the energy, by using Equation (55),

$$
E=\int d w d \Omega n\left(w, T_{o}, \frac{v}{c},\right) \hbar w=\gamma E_{o}\left(1+\frac{v^{2}}{3 c^{2}}\right) .
$$

Apparently, a contradiction appears if we compared it with Equation (7). However, this quantity possesses the bulk energy [18,27,30], which comes from the momentum contribution to the energy of the whole system, and it does not contribute to the thermodynamics of the system. It is necessary to rest it from the energy described in Equation (60). Let us calculate it.

$$
\vec{G}=\int d w d \Omega n\left(w, T_{o}, \frac{v}{c},\right) \frac{\hbar \vec{w}}{c}=\frac{4}{3} \gamma E_{o} \frac{\vec{\nu}}{c^{2}}
$$


The momentum $\vec{G}$ generates the so-called bulk energy or the "infamous" work term $\vec{v} \cdot \vec{G}$, as Requardt called it [27]. Now, the internal energy becomes:

$$
\begin{aligned}
U & =E-\vec{\nu} \cdot \vec{G}=E_{o}\left[\gamma\left(1+\frac{v^{2}}{3 c^{2}}\right)-\frac{4}{3} \gamma \frac{\nu^{2}}{c^{2}}\right] \\
& =\gamma E_{o}\left[1+\frac{v^{2}}{3 c^{2}}-\frac{4}{3} \frac{\nu^{2}}{c^{2}}\right] \\
& =\gamma E_{o}\left[1-\frac{v^{2}}{c^{2}}\right]=\gamma^{-1} E_{o},
\end{aligned}
$$

which corresponds to the proposal done by Ares de Parga et al. [18,21,22]. This shows that the momentum gained by the system viewed from the laboratory reference frame does not contribute to thermodynamical quantities.

The result, in Equation (60), has been obtained by directly calculating the mechanical energy; however, the internal energy must be deduced by using statistical mechanics. This means that the mechanical energy, which is part of a four-energy-momentum, is different from the internal energy, which transforms as the temperature in this model. Therefore, it is necessary to deduce the partition function. The regular internal energy is obtained by calculating the partition function, then obtaining the Hemholtz free energy. From the last thermodynamic potential, the pressure and the entropy can be obtained and, finally, the internal energy. However, we need to calculate the partition function for a moving system. It is known that the partition function for the rest frame can be expressed by [33]:

$$
\ln Z_{p h o} d \Omega d w=-\frac{V_{o} w^{2}}{\pi^{2} c^{3}} \ln \left[1-\exp -\frac{h w}{k T_{o}}\right] d \Omega d w .
$$

Therefore,

$$
F=\int-k T_{o} \ln Z_{p h o} d \Omega d w
$$

Then, since:

$$
P=\left[\frac{\partial F_{o}}{\partial V_{o}}\right]_{T_{o}} \quad \text { and } \quad S=-\left[\frac{\partial F_{o}}{\partial T_{o}}\right]_{V_{o}},
$$

The internal energy can be calculated, and it is equal to:

$$
U_{o}=E_{o}=\frac{3 \xi(4)}{\pi \hbar^{3} c^{3}} V_{o} k^{3} T_{o}^{3} .
$$

Now, following the same procedure used for the particle density for a moving blackbody, we propose:

$$
\ln Z_{p h} d \Omega d w=-\frac{V w^{2}}{\pi^{2} c^{3}} \ln \left[1-\exp -\frac{h w \gamma\left(1-\frac{u}{c} \cos \theta\right)}{k T_{o}}\right] d \Omega d w
$$

Following the same technique, it is obtained:

$$
U=\gamma^{-1} U_{o}=\gamma^{-1} E_{o}
$$

As we expected, statistical physics gives the correct transformation of the thermodynamical quantities, while mechanical definitions, as in the case of the energy, Equation (60), generate incorrect thermodynamical functions that have to be corrected in order to obtain the well-defined quantities in the moving frame as happens in Equation (62), within this theory. 
A covariant theory, congruent with the above results, was developed by Ares de Parga and López-Carrera [21,22], where the choice of the instantaneous volume plays a fundamental role. A volume has to be defined in a reference frame where the measurement of its length can be formally done; that is, by using simultaneity. This was explained since the beginning of special relativity by Fermi [40]. Synge [41], Gamba [23], Nakamura [19,20] and Rohrlich [15,42] recovered Fermi's idea, and a covariant volume was defined. The choice of the volume does not have to correspond to the volume of a system in its rest frame [21,22]. The scalar volume and the four-vector volume are defined by:

$$
V=\frac{V_{o}}{w^{\nu} u_{\nu}}, \quad V^{\mu}=\frac{w^{\mu}}{w^{\nu} u_{\nu}} V_{o},
$$

where $w^{\mu}$ and $u^{\mu}$ represent the unit vector that determines the direction of the three-dimensional volume in the four-dimensional space and the normal vector, which describes the motion between $K$ and $K_{o}$, respectively. As an example of this, if we choose the volume to be measured in the laboratory frame (Einstein-Planck-like proposal) $w^{\mu}=(1,0,0,0)$ and $u^{\mu}=\left(\gamma, \gamma \frac{\vec{v}}{c}\right)$, but if we opt for measuring the volume in the rest frame (Rohrlich-Gamba proposal), $w^{\mu}=\left(\gamma, \gamma \frac{\vec{v}}{c}\right)$ and $u^{\mu}=\left(\gamma, \gamma \frac{\vec{v}}{c}\right)$. Returning to the general case, the entropy is defined by:

$$
d S=\beta_{\mu} \frac{V_{o} w_{\nu}}{u^{\lambda} w_{\lambda}} d T^{\mu \nu}+\beta_{\mu} p d V^{\mu},
$$

where the energy-momentum density tensor $T^{\mu \nu}$ is defined by:

$$
T^{\mu \nu}=\left(p+e_{o}\right) u^{\mu} u^{v}-p g^{\mu \nu},
$$

where $e_{o}$ represent the energy density and:

$$
\beta_{\mu}=\frac{u_{\mu}}{T_{o}}
$$

is the four-inverse temperature. Therefore, without specifying which kind of volume we have chosen and by noticing that $u^{\mu}$ is a unit, we arrive at:

$$
\begin{aligned}
d S & =\beta_{\mu} \frac{V_{o} w_{\nu}}{u^{\lambda} w_{\lambda}} d T^{\mu \nu}+\beta_{\mu} p d V^{\mu} \\
& =\beta_{\mu} \frac{V_{o} w_{\nu}}{u^{\lambda} w_{\lambda}} d\left[\left(p+e_{o}\right) u^{\mu} u^{v}-p g^{\mu \nu}\right]+\beta_{\mu} p d\left[w^{\mu} \frac{V_{o}}{u^{\lambda} w_{\lambda}}\right] \\
& =\frac{1}{T_{o}} d E_{o}+\frac{p}{T_{o}} d V_{o},
\end{aligned}
$$

which shows the invariance of the entropy. In special relativity, mechanical energy must be considered as the zero-component of a four-vector energy-momentum. In this case, the thermodynamical four-energy-momentum can be defined as:

$$
\xi^{\mu}=P^{\mu}-G^{\mu}
$$

where $P^{\mu}$ corresponds to the classical:

$$
P^{\mu}(w)=\int_{V\left[w^{\mu}\right]} T^{\mu v} d V_{\nu}=\frac{V_{o} T^{\mu \nu} w_{\nu}}{u^{\lambda} w_{\lambda}}=T^{\mu \nu} V_{\nu} .
$$


and $G^{\mu}$ represents the generalization of the bulk energy to relativity; that is,

$$
\begin{aligned}
G^{\mu} & =\left[\left(p+e_{o}\right) u^{\mu} u^{\nu}-\left(p+e_{o}\right) g^{\mu \nu}\right] \frac{V_{o} w_{\nu}}{u^{\lambda} w_{\lambda}} \\
& =\left(p V_{o}+E_{o}\right) u^{\mu}-\left(p V_{o}+E_{o}\right) \frac{w^{\mu}}{u^{\lambda} w_{\lambda}} .
\end{aligned}
$$

We construct the thermodynamical four-vector energy-momentum or the four-vector redefined internal energy $\xi^{\mu}$ as:

$$
\begin{aligned}
\xi^{\mu}= & P^{\mu}-G^{\mu} \\
= & \left(p V_{o}+E_{o}\right) u^{\mu}-p V_{o} \frac{w^{\mu}}{u^{\lambda} w_{\lambda}} \\
& -\left(p V_{o}+E_{o}\right) u^{\mu}-\left(p V_{o}+E_{o}\right) \frac{w^{\mu}}{u^{\lambda} w_{\lambda}} \\
= & \frac{w^{\mu}}{u^{\lambda} w_{\lambda}} E_{o} .
\end{aligned}
$$

The first law can be written as:

$$
d \xi^{\mu}=\delta \Theta^{\mu}-d W^{\mu}
$$

where:

$$
d \xi^{\mu}=w^{\mu} \frac{d E_{o}}{u^{\lambda} w_{\lambda}}, \quad \delta \Theta^{\mu}=w^{\mu} \frac{\delta Q_{o}}{u^{\lambda} w_{\lambda}}, \quad \text { and } \quad d W^{\mu}=w^{\mu} \frac{p d V_{o}}{u^{\lambda} w_{\lambda}},
$$

where $\delta \Theta^{\mu}$ and $W^{\mu}$ represent the four-vector heat and the four-vector work, respectively. The entropy can be related to the heat by:

$$
d S=\beta_{\mu} \delta \Theta^{\mu}=\frac{\delta Q_{o}}{T_{o}} .
$$

Equation (78) can be written as:

$$
w^{\mu} \frac{d E_{o}}{u^{\lambda} w_{\lambda}}=w^{\mu} \frac{d Q_{o}}{u^{\lambda} w_{\lambda}}-w^{\mu} \frac{p d V_{o}}{u^{\lambda} w_{\lambda}} .
$$

For the Einstein-Planck model [21,22], we have:

$$
w^{\mu}=(1,0,0,0) \quad \text { and } \quad u^{\mu}=\left(\gamma, \gamma \frac{u}{c}, 0,0\right) ;
$$

that is the volume being measured in the laboratory frame. In this case, Equation (81) is:

$$
(1,0,0,0) \frac{d E_{o}}{\gamma}=(1,0,0,0) \frac{d Q_{o}}{\gamma}-(1,0,0,0) \frac{p d V_{o}}{\gamma},
$$

which corresponds to:

$$
d U=\delta Q-p d V
$$

and it coincides with Equations (5), (7) and (17) if the heat transforms as $\delta Q=\gamma^{-1} \delta Q_{o}$; that is: $a=\gamma^{-1}$.

It has to be noted that different choices of $w^{\mu}$ and $u^{\mu}$ lead to different transformation laws, as has been demonstrated by Ares de Parga and López-Carrera [21,22] or in a different, but very close, theory by Nakamura [19,20] (the $\theta$ and $\phi$ parameters correspond to a choice of $w^{\mu}$ and $u^{\mu}$ ). For example, for the Rohrlich-Gamba proposal [21,22], $w^{\mu}=\left(\gamma, \gamma \frac{\vec{v}}{c}\right)$ and $u^{\mu}=\left(\gamma, \gamma \frac{\vec{v}}{c}\right)$, the transformation of the temperature is $T=\gamma T_{o}$. In this order of ideas, the theory developed by Ares de Parga and 
López-Carrera $[21,22]$ is completely covariant, but the choice of the volume defines the transformation laws, as has been shown by defining a thermometer for the Einstein-Planck theory [21,22]. Other thermometers can be defined depending on the choice of the reference frame where simultaneity is considered. It has to be remembered that it is supposed to be dealing with an equilibrium system (closed system) where thermodynamical quantities do not depend on the time, but the volume has to be defined in a reference frame.

\section{Concluding Remarks}

The Lorentz transformations have been obtained by using the invariances of the laws of thermodynamics and the efficiency of the Carnot cycle in a similar way such that they can be deduced from the invariances of the Maxwell equations and the speed of light.

Ares de Parga et al. obtained the Einstein-Planck theory [18,21,22], with some modifications, by noticing that the internal energy has to be redefined in relativistic thermodynamics. Based on the Nakamura approach, they were able to develop a covariant theory whose fundamental characteristic consists of defining the volume $V_{o}$ in the rest frame, giving a covariant definition of the volume in another frame. In this proposal, the principal result was that the internal energy, $U$, transforms as $U=\gamma^{-1} U_{o}=\gamma^{-1} E_{o}$. By considering that the laws of thermodynamics are invariant, the Ares de Parga et al. proposal is recovered [21,22]. This result supports the idea that considering the invariance of the laws of classical thermodynamics, the consistent theory for the relativistic transformation laws is the Einstein-Planck proposal with the corrections done by Ares de Parga et al. [21,22]. Other theories could be correct, but the definitions of the temperature and the heat differ from the definitions that are consistent considering the invariance of classical thermodynamics. Actually, by supposing the Lorentz transformations with the covariant method assumption, the inverse theory was developed by Ares de Parga et al. [18,21,22].

An important result to be noted is that classical thermodynamics allows other kind of dynamics. Particularly, a compatibility appears in a natural way with general relativity.

\section{Acknowledgments}

This work was partially supported by the following Mexican institutions: COFAA-IPN, EDI-IPN and CONACYT.

\section{Author Contributions}

The authors contributed equally to the paper. All authors have read and approved the final manuscript.

\section{Conflicts of Interest}

The authors declare no conflict of interest. 


\section{References}

1. Einstein, A. Über das Relativitätsprinzip und die aus demselben gezogenen Folgerungen. Jahrbuch der Radioaktivität und Elektronik 1907, 4, 411-462.

2. Planck, M. Zur Dynamik bewegter Systeme. Annalen der Physik 1908, 331, 1-34.

3. Liu, C. Einstein and relativistic thermodynamics in 1952: A historical and critical study of a strange episode in the history of modern physics. Br. J. Hist. Sci. 1992, 25, 185-206.

4. Landsberg, P.T.; Matsas, G.E.A. Laying the ghost of the relativistic temperature transformation. Phys. Lett. A 1996, 223, 401-462.

5. Van Kampen, N.G. Relativistic Thermodynamics of Moving Systems. Phys. Rev. 1968, 173, 295-301.

6. Israel, W. Nonstationary irreversible thermodynamics: A causal relativistic theory. Ann. Phys. 1976, 100, 310-331.

7. Israel, W. Thermodynamics of relativistic systems. Physica A 1981, 106, 204-214.

8. Balescu, R. Relativistic Statistical Thermodynamics. Physica 1968, 40, 309-338.

9. Staruszkiewicz, A. Relativistic transformation laws for thermodynamical variables with applications to classical electron theory. Il Nuovo Cimento A 1966, 45, 684-688.

10. Ott, H. Lorentz-Transformtion der Warme und der Temperatur. Zeitschrift für Physik A 1963, 175, 70-104.

11. Arzeliès, H. Transformation Relativiste de la Temperature et de quelques autres grandeurs Thermodynamiques. Il Nuovo Cimento Ser. 10 1965, 35, 792-804.

12. Möller, C. The Theory of Relativity; International Series of Monographs on Physics; Clarendon Press: Oxford, UK, 1972; Chapter 7.

13. Przanowski, M.; Tosiek, J. Notes on thermodynamics in special relativity. Physica Scripta 2011, 84, doi:10.1088/0031-8949/84/05/055008.

14. Landsberg, P.T. Does a Moving Body Appear Cool? Nature 1966, 212, 571-572.

15. Rohrlich, F. True and apparent transformations, classical electrons, and relativistic thermodynamics. Il Nuovo Cimento B 1966, 45, 76-83.

16. Sieniutycz, S. Thermodynamic and relativistic aspects of thermal inertia in fluids. Phys. Rev. E 1998, 58, 7027-7039.

17. Landsberg, P.T; Matsas, G.E.A. The impossibility of a universal relativistic temperature transformation. Physica A 2004, 340, 92-94.

18. Ares de Parga, G.; López-Carrera, B.; Angulo-Brown, F. A proposal for relativistic transformations in thermodynamics. J. Phys. A 2005, 38, 2821-2834.

19. Nakamura, T.K. Relativistic Energy-Momentum of a Body with a Finite Volume. Space Sci. Rev. 2006, 122, 271-278.

20. Nakamura, T.K. Three Views of a Secret in Relativistic Thermodynamics. Prog. Theor. Phys. 2012, 128, 463-475.

21. Ares de Parga, G.; López-Carrera, B. Relativistic Statistical Mechanics vs. Relativistic Thermodynamics. Entropy 2011, 13, 1664-1693. 
22. Ares de Parga, G.; López-Carrera, B. Redefined relativistic thermodynamics based on the Nakamura formalism. Physica A 2009, 388, 4345-4356.

23. Gamba, A. Physical Quantities in Different Reference Systems According to Relativity. Am. J. Phys. 1967, 35, 83-89.

24. Kibble, T.W.B. Relativistic Transformation Laws for Thermodynamic Variables. Il Nuovo Cimento B 1966, 41, 72-78.

25. Arzeliès, H. Comment on Dr. Kibble's Article. Il Nuovo Cimento B 1966, 41, 81-82.

26. Gamba, A. Remarks to the Preceding Letter by Kibble. Il Nuovo Cimento B 1966, 41, 79-80.

27. Requardt, M. Thermodynamics meets Special Relativity-or what is real in Physics? 2008, arXiv:0801.2639.

28. Noerdlinger, P.D. A Moving Body must "appear" cool. Nature 1967, 213, 1117-1118.

29. Autobiographical Notes (1946), 33. Quoted in Albert Einstein: Historical and Cultural Perspectives; Holton, G., Elkana, Y., Eds.; Dover Publications: Mineola, NY, USA, 1997; p. 227.

30. Tolman, R.C. Relativity Thermodynamics and Cosmology; Oxford University Press: Oxford, UK, 1934; Chapter 5.

31. Angulo-Brown, F. An ecological optimization criterion for finite-time heat engines. J. Appl. Phys. 1991, 69, 7465-7476.

32. Landau, L.D.; Lifshitz, E.M. Statistical Physics; Pergamon Press: London, UK, 1958; Chapters 27 and 60.

33. Greiner, W.; Neise, L.; Stöcker, H. Thermodynamics and Statistical Mechanics; Springer-Verlag: Berlin, Germany, 1995; Chapter 13.

34. Terletskii, Y.P. Paradoxes in the Theory of Relativity; Plenum Publishing: New York, NY, USA, 1968; Section 7.

35. Mermin, N.D. Relativity without light. Am. J. Phys. 1984, 52, 119-124.

36. Henry, G.R.; Feduniak, R.B.; Silver, J.E.; Peterson, M.A. Distribution of Blackbody Cavitty Radiation in a Moving Frame of Reference. Phys. Rev. 1968, 176, 1451-1455.

37. Peebles, P.J.E.; Wilkinson, D.T. Comment on the Anisotropy of the Primeval Fireball. Phys. Rev. 1968, 174, 2168.

38. Bracewell, R.N.; Conklin, E.K. An Observer moving in the $3^{\circ}$ K Radiation Field. Nature 1968, 219, 1343-1344.

39. Jacobson, T. Thermodynamics of Spacetime. Phys. Rev. Lett. 1995, 75, 1260-1263.

40. Segre, E.; Fermi, E. Note e Memorie-Collected Papers; University of Chicago Press: Chicago, IL, USA, 1962; Volume I.

41. Synge, J.L. Relativity, The Special Theory; North-Holland: Amsterdam, The Netherlands, 1956; p. 429.

42. Rohrlich, F. Classical Particles, Foundations of Their Theory; Addison-Wesley: Reading, PA, USA, 1965; p. 282.

(c) 2015 by the authors; licensee MDPI, Basel, Switzerland. This article is an open access article distributed under the terms and conditions of the Creative Commons Attribution license (http://creativecommons.org/licenses/by/4.0/). 